\title{
Modelling inequalities in the availability of National Health Service GDPs to socio-demographic factors
}

\author{
Inequalities in availability of National Health Service general dental practitioners in England and Wales \\ by D. R. Moles, C. Frost, C. Grundy Br Dent J 2001; 190:548-553
}

\begin{abstract}
Aim
To model the inequalities in availability of National Health Service general dental practitioners in England and Wales in relation to key socio-demographic factors.
\end{abstract}

\section{Methods \\ Current estimates of the numbers of NHS general dental practitioners for each health authority were related to data from the 1991 census using Poisson regression models, and generalised estimating equations to allow for correlation between results for neighbouring health authorities.}

\section{Results}

An 'average' health authority, without a dental school, would be expected to have 2,138 residents for every NHS dentist. Controlling for relevant factors, health authorities with higher proportions of the following are associated with lower (better) population to dentist ratios by the amounts shown: each $1 \%$ higher female population $(-11.8 \%$; 95\% CI $-19.1 \%,-3.9 \%$ $P=0.004)$; each $1 \%$ greater South Asian population $(-1.4 \%$; $95 \% \mathrm{CI}-2.1 \%,-0.7 \% P<0.001)$. A health authority with a dental school is associated with a more favourable ratio compared with one without such a facility $(-9.2 \%$; $95 \% \mathrm{CI}-16.2 \%,-1.6 \%$ $P=0.019)$. Each additional $1 \%$ of the following are associated with a worse ratio by the amounts shown: children aged 0 to 14 years old $(+5.2 \%$; $95 \% \mathrm{CI}+2.4 \%,+8.1 \% P<0.001)$; adults aged over 65 years old $(+2.8 \%, 95 \% \mathrm{CI}+1.0 \%,+4.7 \% P=0.002)$; households without a car $(+0.8 \%$; $95 \%$ CI $0.0 \%,+1.6 \% P=0.042)$.

\section{Conclusions}

Ensuring access to dental care may be a more complex issue than simply providing adequate numbers of dentists at a national level. Any manpower planning exercise should additionally consider local factors that may act as incentives or disincentives to those professionals who provide care.

\section{In brief}

- There are geographical inequalities in the numbers of NHS GDPs in England and Wales.

- The inequalities can be predicted using demographic information from the last census.

- Health authorities with higher proportions of women and/or South Asian people are associated with better availability of NHS GDPs.

- Health authorities with a dental school also have better availability of local NHS GDPs.

- These inequalities cannot be addressed without an adequate understanding of the factors that affect where dentists practise.

\section{Comment}

Tncreasing access to NHS dental care and reducing inequalities in oral health are major elements in the Governments strategy for 'Modernising NHS Dentistry - Implementing the NHS Plan'. Whilst much research has been directed at the factors that effect demand for services from consumers of healthcare little has been directed towards the supply side human resource issues. Are the inequalities seen in service uptake mainly associated with local population socio-demographic factors or lack of service supply? Is demand for dental services supplier induced? This interesting and timely paper uses routinely collected data to construct a model to describe and predict the geographical inequalities in avail-
\end{abstract}

ability of NHS general dental practitioners in England and Wales. The authors recognise the problem and limitations associated with using dentist population ratios at health authority level, and of using numbers of dental practitioners in contract with a health authority. None the less they produce some very interesting associations identifying lower availability of practitioners where the proportion of the population are very young or elderly, and lower numbers of people per practitioner in health authorities with greater proportion of females or South Asians.

The impact of the presence of a dental teaching school in a health authority is shown to be considerable, supporting pre- vious work identifying the influence on a practitioner of their alma mater. This reinforces the need for those involved in workforce planning and service provision at all levels to involve such institutions in an imaginative way to influence more equitable distribution in the future. This might be through such approaches as outreach teaching, networking or distance learning and video conferencing whether at undergraduate or postgraduate level or in the education of professionals complementary to dentistry.

\section{E. Gibbons}

Professor, Guy's, King's \& St Thomas Dental Institute 